\title{
Médicos y bioquímicos farmacéuticos en el cuidado personalizado de la salud con la formulación magistral.
}

Doctors and pharmaceutical biochemists in personalized health care with the magistral formulation.

Nelly Ivonne Guananga Diaz. ${ }^{1}$, Valeria Isabel Rodríguez Vinueza. ${ }^{2}$, Verónica Mercedes Cando Brito. ${ }^{3} \&$ Junior Alfredo Escobar Torres. ${ }^{4}$

\section{DOI: https://doi.org/10.33262/anatomiadigital.v4i2.1668}

\begin{abstract}
.
Introduction. The shortage of drugs, the prescription of expensive brand name drugs and the underutilization of the Pharmaceutical Biochemist's knowledge to formulate personalized drugs, reduce quality and health coverage. Objective. To learn the management given to magisterial formulations (MF) by Pharmaceutical Physicians and Biochemists (BQFs). Methodology. Descriptive, cross-sectional study, carried

\section{Resumen.}

Introducción. El desabastecimiento de medicamentos, la prescripción de costosos medicamentos de marca y la infrautilización de los conocimientos del Bioquímico Farmacéutico para formular medicamentos personalizados, aminoran la calidad y cobertura de salud. Objetivo. Conocer el manejo que otorgan a las formulaciones magistrales (FM.) los Médicos y Bioquímicos Farmacéuticos (BQFs). Metodología. Estudio

1 Escuela Superior Politécnica de Chimborazo, Facultad de Ciencias. Grupo de Investigación LEISHPAREC. Riobamba, Ecuador. nguananga@espoch.edu.ec, (D) https://orcid.org/0000-0003-04517878

2 Escuela Superior Politécnica de Chimborazo, Facultad de Ciencias. Grupo de Investigación LEISHPAREC. Riobamba, Ecuador. valeria.rodriguez@espoch.edu.ec, (D) https://orcid.org/0000-00015515-3539

3 Escuela Superior Politécnica de Chimborazo, Facultad de Ciencias. Grupo de Investigación LEISHPAREC. Riobamba, Ecuador. vcando@yahoo.es, (D) https://orcid.org/0000-0002-7353-0227

${ }^{4}$ Escuela Superior Politécnica de Chimborazo, Facultad de Ciencias. Riobamba, Ecuador. alfredoesc29@gmail.com
\end{abstract}


out in Riobamba in May and June 2018 with 101 doctors and 20 BQFs. Variables: knowledge, opinion, intention and application of the MF. Descriptive statistics, Ch2 and Likert scale analysis were obtained. Results. At $\alpha=0.05$, there is no association between types of doctors and study variables. Knowledge: $75 \%$ of doctors insufficient and regular, and $25 \%$ acceptable and excellent; 54\% BQF insufficient and fair, and $46 \%$ acceptable and excellent. Opinion: For doctors, $45.29 \%$ personalization, low costs, dose flexibility and that they cover the shortage of medicines are not advantages, for $54.7 \%$ yes; disadvantages: lack of production premises, obsolete, without sanitary registration, and they are not applicable to all specialties, for $47 \%$ they are not disadvantages, for $53 \%$ they are; BQFs agree more with the advantages and less with the disadvantages. Application: $59.3 \%$ doctors prescribe very little. The BQFs: $45 \%$ elaborate very little, $80 \%$ indicate that they are prepared to elaborate them and $100 \%$ consider them a job opportunity; meanwhile, for $43 \%$ of doctors there are no professionals who prepare MF. Intention: $81.7 \%$ doctors and 97.5\% BQFs wish to expand their knowledge and attend updating programs. Conclusion. Doctors prescribe very little, have little knowledge to do so, and are unaware of quality control and the variety of magisterial formulas. Most of the BQFs do not prepare them and have a lack of knowledge, especially about pharmacopoeias and legislation. Most of the patients never ask for magistral formulas.

Keywords: Magisterial Formulation, prescription, quality control, pharmacopoeia. descriptivo, transversal, realizado en Riobamba en mayo y junio del 2018 a 101 médicos y 20 BQFs. Variables: conocimiento, opinión, intención y aplicación sobre la FM. Se obtuvieron estadísticos descriptivos, $\mathrm{Ch}^{2}$ y análisis de escalas Likert. Resultados. A $\alpha=0,05$ no hay asociación entre tipos de médicos y variables de estudio. Conocimiento: $75 \%$ de médicos insuficiente y regular, y $25 \%$ aceptables y excelentes; 54\% BQFs insuficiente y regular, y $46 \%$ aceptable y excelente. Opinión: Para médicos, el $45,29 \%$ no son ventajas la personalización, bajos costos, flexibilidad de dosis y que cubren el desabastecimiento de medicamentos, para el 54,7\% si; desventajas: falta de locales de elaboración, obsoletas, sin registro sanitario, y no son aplicables a toda especialidad, para el $47 \%$ no son desventajas, para el 53\% si; los BQF están más de acuerdo con las ventajas y menos de acuerdo con las desventajas. Aplicación: 59,3\% médicos prescriben muy poco. Los BQFs: $45 \%$ elaboran muy poco, $80 \%$ indican estar preparados para elaborarlas y $100 \%$ las considera una oportunidad de trabajo; mientras, para el $43 \%$ de los médicos no hay profesionales que elaboren FM. Intención: 81,7\% médicos y 97,5\% BQFs desean ampliar conocimientos y asistir a programas de actualización. Conclusión. Los médicos prescriben muy poco, presentan bajo conocimiento para hacerlo, y desconocen sobre el control de calidad y variedad de fórmulas magistrales. Los BQFs en su mayoría no las elaboran y tienen deficiencia en conocimientos especialmente sobre farmacopeas $y$ legislación. Los pacientes en su mayoría nunca solicitan fórmulas magistrales. 
Palabras claves: Formulación magistral, prescripción, control de calidad, farmacopea.

\section{Introducción.}

La aparición de la formulación magistral prácticamente nace con la humanidad, hallando en la naturaleza la fuente de sus remedios para tratar sus problemas de salud, experimentando con sustancias naturales provenientes de plantas (Garcia \& Molinero, 2014) y otras fuentes, de manera que se desarrollaron diversos procedimientos y fórmulas que fueron la base de la alquimia, evolucionando hacia la farmacia moderna y los componentes de los actuales medicamentos.

A la par aparecen los preparados y la figura del farmacéutico, así, en la leyenda griega Higía era el boticario o farmacéutico. Una de las clases de los médicos-sacerdotes de Egipto se encargaban de elaborar los remedios en los templos, los herbolarios de la Edad Media en Europa y la antigua Grecia eran quienes facilitaban las materias primas a los médicos. En 1683 el ayuntamiento de Brujas prohibió a los médicos preparar medicamentos para sus pacientes. En América, Benjamín Franklin separa al médico del farmacéutico al nombrar un boticario para el Hospital de Pensilvania (Godínez, Aceves, \& Schifter, 2019). Los farmacéuticos y químicos árabes de la época medieval elaboraron medicamentos compuestos, y llegaron a abrir las primeras farmacias en Bagdad en el año 754 A.C. (Zebroski, 2016).

Las fórmulas magistrales fueron los únicos medicamentos disponibles, e incluso a mediados de la pasada centuria suponían el $60 \%$ de las prescripciones médicas. El desarrollo de la industria de fármacos, desplazo a la formulación, sin embargo, su preparación y uso individualizado tienen ventajas sobre los de fabricación industrial (Ortiz de Zarate, 2016), otra ventaja fue introducir la farmacia en los estudios universitarios, lo cual otorgo mayor rigor científico, profesionales farmacéuticos mejor preparados y promovió la apertura de un creciente número de farmacias (Lamberts, et al., 2021). El farmacéutico es reconocido como un elemento importante en el equipo de salud, ya sea en la farmacia comunitaria u hospitalaria, teniendo un papel significativo en la seguridad de la medicación del paciente, se han reconocido las mejores escuelas de farmacia del mundo y los países con la mejor formación de farmacéuticos, destacando varias universidades de Estados Unidos, como la Universidad de California en San Francisco, así como varias universidades en el Reino Unido, como la Universidad de Cambridge, Oxford y otros. Australia también ha sido mencionada como líder en educación farmacéutica, y algunas universidades en Suecia y España (Rankings, 2020).

El médico debe contar con el adecuado nivel de conocimientos sobre formulaciones magistrales, para posterior al diagnóstico y evaluación del paciente, prescriba acorde a las especiales e individuales necesidades del paciente, y contribuya además para que estas medicinas no estén infrautilizadas (Fernández \& Bustamante, 2020). En 2010 se reportó en España una demanda de FM entre el 0,5-2\% de las dispensaciones de las oficinas de 
farmacia, en algunos países de Europa representaba el 3\% (Ruiz, 2015). En otros países el empleo de estos medicamentos ha ido en crecimiento, como en Cuba, España, Canadá, y otros (Rodríguez, 2012; Zúñiga, 2016).

Ecuador es uno de los países sudamericanos con mayor consumo de medicamentos per cápita, sus empresas generan millones de dólares en fármacos de marca registrada, mientras los medicamentos genéricos con muy consumidos, por lo tanto el derecho de salud es fácilmente vulnerado (Vite-Vera \& Párraga-Fernández, 2019), la importación de medicamentos constituye el 80\% (OPS/OMS, 2012), el consumidor no está al tanto de la ley por lo que las cadenas de farmacias ofrecen el producto más caro. En el Hospital Dermatológico Gonzalo González se encontró una demanda insatisfecha del 85\% de la prescripción y se señala que la alternativa de aprovisionamiento para medicamentos tópicos constituye la formulación magistral y oficinal, con las claras ventajas de dosificación personalizada, ahorro y adherencia al tratamiento, sumándose la dispensación ejercida por los profesionales farmacéuticos dentro del ejercicio de la atención farmacéutica especializada (Sacoto, 2016).

En Riobamba, la Botica Bristol es el único local que ofrece medicamentos industrializados y FM, en el área de la formulación cuenta con 260 preparados que cumplen estándares de calidad y seguridad, anualmente preparan unas 1.200 formulaciones tanto para los médicos de la ciudad como para los de otras ciudades del país (El Telégrafo, 2017). Las características de estos medicamentos como el precio, calidad, y confianza en la resolución de dolencias individuales y de enfermedades especiales hacen que sigan siendo demandadas (Botica Bristol, 2017). Sin embargo, esta botica no abastece para la variedad de especialidades, la pequeña oferta en preparados individualizados y ausencia de investigación en esta área lleva a los médicos a buscar FM en otras ciudades del país (Fernández \& Bustamante, 2020). En los países en desarrollo los farmacéuticos todavía están infrautilizados y su papel como profesionales de la salud no se considera importante ni por la comunidad ni por otros proveedores de atención de la salud. La interrelación médico-farmacéutico-paciente es primaria para identificar la necesidad de cierto paciente y elaborar los medicamentos magistrales que atiendan su derecho a una atención sanitaria segura y efectiva, los pacientes denominados con enfermedades y problemas de salud especiales suelen recibir escasa atención terapéutica, ya que la industria farmacéutica es reacia a desarrollar medicamentos para estos casos por su baja rentabilidad, es cuando la personalización de las fórmulas magistrales es de gran relevancia (Corral, 2006).

Esta investigación busca conocer la importancia y el manejo que otorgan a las formulaciones magistrales los Bioquímicos Farmacéuticos y los Médicos de la ciudad de Riobamba, información que se obtiene con la aplicación de encuestas dirigidas a evaluar el conocimiento, opinión, aplicación e intención respecto a estos medicamentos, con el fin de acercarnos a la realidad sobre el rol que están desempeñando estos profesionales en el sistema de atención de la salud actual de la ciudad. 


\section{Metodología.}

\section{Localización geográfica y temporal}

La investigación se realizó en la ciudad de Riobamba, provincia de Chimborazo, entre los meses mayo y junio del 2018, en diferentes instituciones: Escuela Superior Politécnica de Chimborazo (ESPOCH), Hospital General Riobamba IESS, Hospital Pediátrico Alfonso Villagómez Román y Hospital Básico Andino de Riobamba, Farmacias Bristol y RioPharma.

\section{Materiales y equipos}

Encuestas, hojas de registros, base de datos de participantes, computador, celular y el software estadístico SPSS 25.

\section{Metodología}

Estudio descriptivo, transversal, analítico, con un total de 121 encuestados distribuidos en: 101 médicos del Hospital General Riobamba IESS (H-IESS), Hospital Pediátrico Alfonso Villagómez Román (HPAVR) y Hospital Universitario Andino de la Universidad Nacional de Chimborazo de Riobamba (HUA-UNACH); 20 BQF de los hospitales mencionados y además de las farmacias Bristol y RioPharma.

Inicialmente se establecieron los objetivos y definieron las variables: conocimiento, opinión, intención y aplicación respecto al tema de la formulación magistral, luego se diseñó el cuestionario en base a las variables indicadas, se aplicó una encuesta piloto constituida por 13 médicos docentes de la ESPOCH, y 13 bioquímicos farmacéuticos del Hospital General Riobamba IESS, la consistencia interna de esta encuesta se determinó con el Índice Alfa Cronbach, parámetro estadístico que permitió acreditar este instrumento. Una vez establecida la encuesta definitiva, se inició el trabajo de campo.

Para calcular el tamaño de la muestra en cada grupo y asegurar la credibilidad de los resultados se empleó la fórmula para poblaciones finitas (Bernal, 2016):

$$
n=\frac{\mathrm{N} \times Z^{2} \times \mathrm{p} \times \mathrm{q}}{(\mathrm{N}-1) \times e^{2}+Z^{2} \times \mathrm{p} \times \mathrm{q}}
$$

En donde:

$\mathrm{N}$ : tamaño de la población, constituye el número total de posibles encuestados.

$\mathrm{Z}$ : constante que indica la probabilidad de que los resultados de la investigación sean reales.

Para $Z=1.96$, nivel de confianza del 95\%.

e: error muestral esperado.

p: proporción de elementos que posee la característica de interés.

q: proporción de individuos que no poseen esa característica de interés.

n: tamaño de la muestra (número de encuestas a realizar) (Bernal, 2016). 
Tabla 1: Población y muestra de Médicos.

\begin{tabular}{ccccc}
\hline Hospitales encuestados & Médicos & Población & $\begin{array}{c}\text { Porcentaje } \\
\text { de médicos }\end{array}$ & $\begin{array}{c}\text { Muestra a } \\
\text { encuestar }\end{array}$ \\
\hline & Médicos especialistas: & 76 & $32,5 \%$ & 33 \\
Hospital General Riobamba & Médicos residentes: & 54 & $23,1 \%$ & 12 \\
IESS (H-IESS) & Médicos generales: & 29 & $12,4 \%$ & 23 \\
& Odontólogos: & 8 & $3,4 \%$ & 3 \\
Hospital Pediátrico Alfonso & Médicos especialistas: & 7 & $3,0 \%$ & 3 \\
Villagómez Román (HPAVR) & Médicos residentes: & 13 & $5,6 \%$ & 6 \\
Hospital General Andino de & Oédontólogos: & 4 & $1,7 \%$ & 2 \\
Chimborazo (HUA-UNACH) & Médicos residentes: & 37 & $15,9 \%$ & 16 \\
Población total de médicos: & 8 & $3.4 \%$ & 3 \\
\hline
\end{tabular}

Fuente: Encuesta aplicada en el H-IESS, HUA-UNACH, y HPAVR

Realizado por: Grupo de Investigación.

Tabla 2: Población y muestra de BQF.

\begin{tabular}{ccc}
\hline Bioquímicos farmacéuticos & Población & Muestra \\
\hline H- IESS & 13 & 12 \\
HPAVR & 3 & 2 \\
HUA-UNACH & 3 & 3 \\
Botica Bristol & 1 & 1 \\
Farmacia RioPharma & 2 & 2 \\
Total & $\mathbf{2 2}$ & $\mathbf{2 0}$ \\
\hline
\end{tabular}

Fuente: Encuesta aplicada en el H-IESS, HUA-UNACH, HPAVR, Farmacias Bristol y RioPharma. Realizado por: Grupo de Investigación

Muestreo: Para el caso los BQF se empleó el muestreo aleatorio al azar y se seleccionó con la técnica de la tómbola. Se aplicó el muestreo por conveniencia para el grupo de médicos de acuerdo a su disponibilidad, aceptación a desarrollar el cuestionario.

\section{Hipótesis:}

Ho: Las variables conocimientos, opinión, aplicación, e intención sobre la formulación magistral son independientes del tipo de médico.

H1: Las variables conocimientos, opinión, aplicación, e intención sobre la formulación magistral no son independientes del tipo de médico.

\section{Análisis Estadístico}

Consistió en los siguientes pasos:

- Tabulación de los datos obtenidos en las encuestas según el grupo: Médicos: generales, especialistas y odontólogos y Bioquímicos Farmacéuticos

- Determinación de los estadísticos descriptivos: frecuencias y tablas de contingencia.

- Establecimiento del estadístico $\mathrm{Chi}^{2}$ para identificar asociación entre variables nominales (tipo de médico) y variables ordinales (conocimiento, opinión, intención y aplicación), en caso de asociación se realizan medidas simétricas y medidas direccionales. 
- Análisis de escalas Likert de las encuestas a médicos y bioquímicos farmacéuticos, para agrupar cada variable en niveles y facilitar la presentación de resultados y análisis.

\section{Resultados.}

De la encuesta piloto

Tabla 3. Resultado del Alfa Cronbach de las encuestas a Médicos y

\begin{tabular}{cc}
$\mathrm{BQF}$. & \\
\hline Muestra piloto encuestada & Alfa de Cronbach \\
\hline Médicos & 0,922 \\
Bioquímicos farmacéuticos & 0,963
\end{tabular}

Fuente: Encuesta aplicada en el H-IESS, HUA-UNACH, HPAVR, Farmacias Bristol y RioPharma. Realizado por: Grupo de Investigación

Los valores del alfa de Cronbach de 0,922 en médicos, y 0,963 en BQF son superiores al valor mínimo de aceptación de 0,80 , están muy cercanos a valor óptimo de 1 , se concluye que las escalas Likert empleadas son confiables, por lo tanto, el cuestionario es válido.

\section{- De las encuestas a médicos}

Tabla 4: Tabla cruzada de tipos de médicos, sexo y rango de edad

\begin{tabular}{|c|c|c|c|c|c|c|c|c|c|}
\hline \multicolumn{6}{|c|}{ Sexo } & \multicolumn{4}{|c|}{ Sexo } \\
\hline & Edad & $\mathbf{M}^{*}$ & $\mathbf{F}_{* *}^{*}$ & Total & & Edad & $\mathbf{M}^{*}$ & $\mathbf{F}^{* * *}$ & Total \\
\hline \multicolumn{10}{|c|}{ Médicos Especialistas } \\
\hline \multirow[t]{5}{*}{ Pediatría } & $30-35$ & 2 & 1 & 3 & Reumatología & 70 & 1 & & 1 \\
\hline & $36-40$ & 1 & 0 & 1 & Oncología & $51-55$ & & 1 & 1 \\
\hline & $41-45$ & 1 & 1 & 2 & Ginecología y Obstetricia & $36-40$ & 1 & 1 & 2 \\
\hline & $46-50$ & 1 & 0 & 1 & & $41-45$ & 0 & 2 & 2 \\
\hline & $51-55$ & 0 & 1 & 1 & & $51-55$ & 0 & 1 & 1 \\
\hline Fisiatría & $51-55$ & 1 & & 1 & Medicina Familiar & $36-40$ & 1 & 0 & 1 \\
\hline \multirow{2}{*}{ Urología } & $41-45$ & 1 & & 1 & & $41-45$ & 1 & 0 & 1 \\
\hline & $56-62$ & 2 & & 2 & & $51-55$ & 0 & 1 & 1 \\
\hline \multirow[t]{4}{*}{ Medicina Interna } & $30-35$ & 0 & 1 & 1 & Medicina Ocupacional & $30-35$ & & 1 & 1 \\
\hline & $41-45$ & 0 & 1 & 1 & Otorrinolaringología & $46-50$ & & 1 & 1 \\
\hline & $46-50$ & 1 & 0 & 1 & Gastroenterología & $41-45$ & & 1 & 1 \\
\hline & $56-62$ & 2 & 0 & 2 & Psiquiatría & $30-35$ & 0 & 1 & 1 \\
\hline Oftalmología & $36-40$ & 1 & 1 & 2 & & $41-45$ & 1 & 0 & 1 \\
\hline \multirow{3}{*}{ Cirugía General } & $30-35$ & 1 & & 1 & Cirugía Vascular & $51-55$ & 1 & & 1 \\
\hline & $41-45$ & 2 & & 2 & & $56-62$ & 1 & & 1 \\
\hline & $46-50$ & 2 & & 2 & Cardiología & $36-40$ & 1 & & 1 \\
\hline Endocrinología & $51-55$ & & 1 & 1 & & 70 & 1 & & 1 \\
\hline \multirow[t]{4}{*}{ Ortopedia y traumatología } & $41-45$ & 1 & & 1 & Terapia Intensiva & $30-35$ & & 1 & 1 \\
\hline & $46-50$ & 1 & & 1 & Neurología & $41-45$ & 1 & & 1 \\
\hline & $56-62$ & 1 & & 1 & Cirugía Pediátrica & $41-45$ & & 1 & 1 \\
\hline & $46-50$ & 1 & & 1 & Geriatría & $41-45$ & 1 & & 1 \\
\hline Total & & & & 29 & \multirow{2}{*}{\multicolumn{5}{|c|}{$\begin{array}{l}\text { Total } \\
\quad \text { Odontólogos }\end{array}$}} \\
\hline \multicolumn{5}{|c|}{ Médicos Generales } & & & & & \\
\hline & $25-30$ & 11 & 16 & 27 & & 27 & 0 & 1 & 1 \\
\hline & $31-35$ & 4 & 8 & 12 & & 28 & 0 & 1 & 1 \\
\hline & $36-37$ & 1 & 3 & 4 & & 32 & 0 & 1 & 1 \\
\hline & 57 & 1 & 0 & 1 & & 37 & 1 & 0 & 1 \\
\hline & & & & & & 65 & 1 & 0 & 1 \\
\hline Total & & 17 & 27 & 44 & Total & & 2 & 3 & 5 \\
\hline
\end{tabular}


De los 52 (100\%) Médicos Especialistas (me) encuestados, 33 (63\%) fueron hombres y $19(36 \%)$ mujeres, especializados en diferentes áreas, siendo los más numerosos en Pediatría, Cirugía General, Ginecología y Obstetricia. De los 44 (100\%) Médicos Generales (mg), se contó con 17 (38,6\%) hombres y 27 (61,3\%) mujeres, este grupo puede apreciarse de edades menores al grupo de Médicos Especialistas. Del grupo de Odontólogos (od) sus edades se encontraron entre 27 y 65 años distribuidos uniformemente en este rango (Tabla 4).

Tabla 5: Resumen de las tablas de contingencias de la variable CONOCIMIENTOS sobre Formulación magistral y tipo de médico.

\begin{tabular}{|c|c|c|c|c|c|c|c|c|}
\hline \multirow{3}{*}{$\begin{array}{l}\text { Escala de } \\
\text { Likert }\end{array}$} & \multicolumn{6}{|c|}{ Sección: Conocimientos } & \multirow{3}{*}{$\begin{array}{l}\text { Chi }^{2} \\
\text { Calc. }\end{array}$} & \multirow{3}{*}{$\begin{array}{l}\text { Signif. asint. } \\
\text { (bilateral) }\end{array}$} \\
\hline & \multicolumn{2}{|c|}{ Médicos especialistas } & \multicolumn{2}{|c|}{ Médicos generales } & \multicolumn{2}{|c|}{ Odontólogos } & & \\
\hline & Frecuencia & $\%$ & Frecuencia & $\%$ & Frecuencia & $\%$ & & \\
\hline \multicolumn{9}{|c|}{ ¿Cuánto considera que conoce sobre formulación magistral? } \\
\hline Insuficiente & 6 & $12 \%$ & 9 & $21 \%$ & & & 9,389 & 052 \\
\hline Regular & 23 & $44 \%$ & 27 & $61 \%$ & 4 & $80 \%$ & gl 4 & \\
\hline Aceptable & 23 & $44 \%$ & 8 & $18 \%$ & 1 & $20 \%$ & & \\
\hline Excelente & 0 & 0 & 0 & 0 & 0 & 0 & & \\
\hline \multicolumn{9}{|c|}{ ¿Cómo calificaría la información sobre formulación magistral recibida en su formación de pregrado? } \\
\hline Insuficiente & 17 & $33 \%$ & 18 & $41 \%$ & 2 & $40 \%$ & 5,677 & ,460 \\
\hline Regular & 31 & $59 \%$ & 19 & $43 \%$ & 3 & $60 \%$ & gl 6 & \\
\hline Aceptable & 3 & $6 \%$ & 7 & $16 \%$ & & & & \\
\hline Excelente & 1 & $2 \%$ & & & & & & \\
\hline \multicolumn{9}{|c|}{ ¿Cómo calificaría la información sobre formulación magistral recibida en su formación de especialización? } \\
\hline Insuficiente & 20 & $38 \%$ & & & 2 & $40 \%$ & 2,623 & ,464 \\
\hline Regular & 19 & $37 \%$ & & & 1 & $20 \%$ & gl 3 & \\
\hline Aceptable & 11 & $21 \%$ & & & 1 & $20 \%$ & & \\
\hline Excelente & 2 & $4 \%$ & & & 1 & $20 \%$ & & \\
\hline \multicolumn{9}{|c|}{ ¿Cuánto conoce sobre la legislación de fórmulas magistrales? } \\
\hline Insuficiente & 23 & $44 \%$ & 29 & $66 \%$ & 1 & $20 \%$ & & ,161 \\
\hline Regular & 21 & $40 \%$ & 11 & $25 \%$ & 2 & $40 \%$ & 9.234 & \\
\hline Aceptable & 7 & $14 \%$ & 4 & $9 \%$ & 2 & $40 \%$ & gl 6 & \\
\hline Excelente & 1 & $2 \%$ & & & & & & \\
\hline \multicolumn{9}{|c|}{ ¿Cuánto conoce sobre algún formulario nacional? } \\
\hline Insuficiente & 17 & $32 \%$ & 16 & $36 \%$ & 2 & $40 \%$ & 8,349 &, 214 \\
\hline Regular & 17 & $33 \%$ & 23 & $52 \%$ & 2 & $40 \%$ & gl 6 & \\
\hline Aceptable & 16 & $31 \%$ & 5 & $12 \%$ & 1 & $20 \%$ & & \\
\hline Excelente & 2 & $4 \%$ & & & & & & \\
\hline \multicolumn{9}{|c|}{ ¿Cuál es su conocimiento sobre los locales a nivel nacional donde se elaboran fórmulas magistrales? } \\
\hline Insuficiente & 13 & $25 \%$ & 14 & $32 \%$ & 1 & $20 \%$ & 7,665 & ,264 \\
\hline Regular & 22 & $42 \%$ & 15 & $34 \%$ & 2 & $40 \%$ & gl 6 & \\
\hline Aceptable & 15 & $29 \%$ & 15 & $42 \%$ & 1 & $20 \%$ & & \\
\hline Excelente & 2 & $4 \%$ & & & 1 & $20 \%$ & & \\
\hline \multicolumn{9}{|c|}{ ¿Cuál es su conocimiento sobre los locales de Riobamba donde se elaboran fórmulas magistrales? } \\
\hline Insuficiente & 13 & $25 \%$ & 10 & $22 \%$ & 1 & $20 \%$ & 6,736 & ,346 \\
\hline Regular & 21 & $40 \%$ & 17 & $39 \%$ & 2 & $40 \%$ & Gl 6 & \\
\hline Aceptable & 15 & $29 \%$ & 17 & $39 \%$ & 2 & $40 \%$ & & \\
\hline Excelente & 3 & $6 \%$ & & & & & & \\
\hline
\end{tabular}

Fuente: H-IESS, HUA-UNACH, y HPAVR

Realizado por: Grupo de Investigación

Los resultados de la variable conocimientos sobre FM (Tabla 5), los mayores porcentajes en los tres grupos de médicos, están distribuidos en las opciones insuficiente y regular, así por ejemplo sobre ¿cuánto consideran conocer sobre formulación magistral?: el 12\% y $21 \%$ de me y mg califican de insuficiente; el $44 \%, 61 \%$ y $80 \%$ de los me, mg y od califican de regular; el 44\%, $18 \%$ y $20 \%$ señalan de aceptable. Respecto a la valoración de la información recibida en su pregrado: el 92\%, 84\% y $100 \%$ de los médicos califican de insuficiente y regular; y $6 \%$ y $16 \%$ de me y mg de aceptable, y apenas el $2 \%$ de me califican de excelente. Agrupando las escalas insuficientes y regular, se tiene: sobre información recibida en estudios de especialización se hallan el 75\% y $60 \%$ de me y od. 
En el orden de me, mg y od, sobre el conocimiento de la legislación $84 \%$, $91 \%$ y $60 \%$; acerca del conocimiento de locales nacionales donde se elaboran fórmulas magistrales el $67 \%, 66 \%$ y $60 \%$; de locales en Riobamba donde se elaboran FM. 65\%, 61\% y 60\%. Los resultados del estadístico $\mathrm{Ch}^{2}$ a un nivel de confianza $\alpha=0,05$ de la variable conocimientos nos indican que no hay asociación entre las variables en estudio.

Para la variable OPINION (Tabla 6), la información se obtuvo a través de siete preguntas que se detallan en la tabla 6 , el $2 \%$ de mg están muy en desacuerdo en considerar importante el uso de FM, el 8\% de me están en desacuerdo, y están indecisos en su opinión el $9 \%$ y $7 \%$ de médicos especialistas y generales. Con un total alto del $83 \%, 91 \%$ y $100 \%$ los me, mg y od están de acuerdo y muy de acuerdo en considerar importante el uso de FM. La prueba $\mathrm{Ch}^{2}$ de la variable opinión a un nivel de confianza $\alpha=0,05$ indica no asociación entre las variables.

Tabla 6: Resumen de las tablas de contingencias de la variable OPINION sobre Formulación magistral y tipo de médico.

\begin{tabular}{|c|c|c|c|c|c|c|c|c|}
\hline \multirow{3}{*}{ Escala de Likert } & \multicolumn{6}{|c|}{ Sección: Opinión } & \multirow{3}{*}{$\begin{array}{l}\text { Chi }^{2} \\
\text { Calc. }\end{array}$} & \multirow{3}{*}{$\begin{array}{l}\text { Signif. asin } \\
\text { Bilateral }\end{array}$} \\
\hline & \multicolumn{2}{|c|}{ Médicos especialistas } & \multicolumn{2}{|c|}{ Médicos generales } & \multirow{2}{*}{$\begin{array}{l}\text { Odontólogos } \\
\text { Frecuencia }\end{array}$} & \multirow[b]{2}{*}{$\%$} & & \\
\hline & Frecuencia & $\%$ & Frecuencia & $\%$ & & & & \\
\hline \multicolumn{9}{|c|}{ ¿Considera importante el uso de fórmulas magistrales? } \\
\hline Muy en desacuerdo & 0 & 0 & 1 & $2 \%$ & 0 & $0 \%$ & 8,538 & ,383 \\
\hline En desacuerdo & 4 & $8 \%$ & 0 & $0 \%$ & 0 & $0 \%$ & $\mathrm{gl} 8$ & \\
\hline Indeciso & 5 & $9 \%$ & 3 & $7 \%$ & 0 & $0 \%$ & & \\
\hline De acuerdo & 30 & $58 \%$ & 33 & $75 \% \quad 16 \%$ & 3 & $60 \%$ & & \\
\hline Muy de acuerdo & 13 & $25 \%$ & 7 & & 2 & $40 \%$ & & \\
\hline \multicolumn{9}{|c|}{ ¿Su especialidad médica requiere de fórmulas magistrales? } \\
\hline Muy en desacuerdo & 4 & $8 \%$ & & & 0 & $0 \%$ & 2,320 & 667 \\
\hline En desacuerdo & 7 & $13 \%$ & & & 0 & $0 \%$ & $\mathrm{gl} 4$ & \\
\hline Indeciso & 5 & $10 \%$ & & & 0 & $0 \%$ & & \\
\hline De acuerdo & 26 & $50 \%$ & & & 4 & $80 \%$ & & \\
\hline Muy de acuerdo & 10 & $19 \%$ & & & 1 & $20 \%$ & & \\
\hline \multicolumn{9}{|c|}{ ¿Considera anticuado el uso de formulaciones magistrales? } \\
\hline Muy en desacuerdo & 13 & $25 \%$ & 9 & $20 \%$ & 0 & $0 \%$ & 8,246 & ,410 \\
\hline En desacuerdo & 22 & $42 \%$ & 20 & $46 \%$ & 4 & $80 \%$ & gl & \\
\hline Indeciso & 4 & $8 \%$ & 8 & $18 \%$ & 0 & $0 \%$ & 8 & \\
\hline De acuerdo & 8 & $15 \%$ & 6 & $14 \%$ & 1 & $20 \%$ & & \\
\hline Muy de acuerdo & 5 & $10 \%$ & 1 & $2 \%$ & 0 & $0 \%$ & & \\
\hline \multicolumn{9}{|c|}{ ¿Los resultados del uso de fórmulas magistrales son buenos? } \\
\hline Muy en desacuerdo & 0 & 0 & 0 & 0 & 0 & $0 \%$ & 8,318 &, 216 \\
\hline En desacuerdo & 4 & $8 \%$ & 2 & $5 \%$ & 0 & $0 \%$ & gl 6 & \\
\hline Indeciso & 14 & $27 \%$ & 17 & $38 \%$ & 0 & $0 \%$ & & \\
\hline De acuerdo & 24 & $46 \%$ & 22 & $50 \%$ & 3 & $60 \%$ & & \\
\hline Muy de acuerdo & 10 & $19 \%$ & 3 & $7 \%$ & 2 & $40 \%$ & & \\
\hline \multicolumn{9}{|c|}{ ¿No hay profesionales que se encarguen de elaborar fórmulas magistrales? } \\
\hline Muy en desacuerdo & 3 & $6 \%$ & 1 & $2 \%$ & 0 & $0 \%$ & 11,506 & ,175 \\
\hline En desacuerdo & 9 & $17 \%$ & 2 & $5 \%$ & 0 & $0 \%$ & g1 8 & \\
\hline Indeciso & 17 & $33 \%$ & 23 & $52 \%$ & 2 & $40 \%$ & & \\
\hline De acuerdo & 19 & $36 \%$ & 11 & $25 \%$ & 3 & $60 \%$ & & \\
\hline Muy de acuerdo & 4 & $8 \%$ & 7 & $16 \%$ & 0 & $0 \%$ & & \\
\hline \multicolumn{9}{|c|}{ Señale las que considere Ventajas de la formulación magistral } \\
\hline & $\mathrm{NO}$ & SI & $\mathrm{NO}$ & SI & NO & SI & & \\
\hline Personalización de los & 13 & 39 & 14 & 30 & 0 & 5 & 2,485 & ,289 \\
\hline tratamientos. & $25 \%$ & & $32 \%$ & $68 \%$ & $0 \%$ & $100 \%$ & $\mathrm{gl} 2$ & \\
\hline \multirow{3}{*}{ Bajos costos. } & & $75 \%$ & & & & & & \\
\hline & 29 & & 24 & 20 & 2 & 3 & ,458 & ,795 \\
\hline & $56 \%$ & $\begin{array}{c}23 \\
44 \%\end{array}$ & $54,5 \%$ & $45,5 \%$ & $40 \%$ & $60 \%$ & $\mathrm{gl} 2$ & \\
\hline \multirow[t]{2}{*}{ Flexibilidad de dosis } & 15 & & 22 & 22 & 3 & 2 & 5,374 & ,068 \\
\hline & $28 \%$ & 37 & $50 \%$ & $50 \%$ & $60 \%$ & $40 \%$ & gl 2 & \\
\hline \multirow{3}{*}{$\begin{array}{c}\text { Cubren desabastecimientc } \\
\text { de medicamentos }\end{array}$} & & $71 \%$ & & & & & & \\
\hline & 33 & & 25 & 19 & 3 & 2 & ,440 & ,802 \\
\hline & $53,5 \%$ & $\begin{array}{c}19 \\
36,5 \%\end{array}$ & $57 \%$ & $43 \%$ & $60 \%$ & 40 & gl 2 & \\
\hline \multicolumn{9}{|c|}{ Señale las que considere Desventajas de la formulación magistral } \\
\hline & NO & SI & NO & SI & NO & SI & & \\
\hline
\end{tabular}




\begin{tabular}{|c|c|c|c|c|c|c|c|c|}
\hline $\begin{array}{l}\text { Falta de lugares de } \\
\text { elaboración. }\end{array}$ & $\begin{array}{c}9 \\
17 \%\end{array}$ & $\begin{array}{c}43 \\
83 \%\end{array}$ & $\begin{array}{c}11 \\
25 \%\end{array}$ & $\begin{array}{c}33 \\
75 \%\end{array}$ & $\begin{array}{c}0 \\
0 \%\end{array}$ & $\begin{array}{c}5 \\
100 \%\end{array}$ & $\begin{array}{c}2,187 \\
\text { gl } 2\end{array}$ &, 335 \\
\hline Han quedado obsoletos. & $\begin{array}{c}35 \\
67 \%\end{array}$ & 17 & $\begin{array}{c}35 \\
80 \%\end{array}$ & $\begin{array}{c}9 \\
20 \%\end{array}$ & $\begin{array}{c}4 \\
80 \%\end{array}$ & $\begin{array}{c}1 \\
20 \%\end{array}$ & $\begin{array}{c}1,944 \\
\text { gl } 2\end{array}$ &, 378 \\
\hline $\begin{array}{c}\text { No poseen registro } \\
\text { sanitario. }\end{array}$ & $\begin{array}{c}30 \\
58 \%\end{array}$ & $33 \%$ & $\begin{array}{c}22 \\
50 \%\end{array}$ & $\begin{array}{c}22 \\
50 \%\end{array}$ & $\begin{array}{c}2 \\
40 \%\end{array}$ & $\begin{array}{l}3 \\
60 \%\end{array}$ & $\begin{array}{c}0,950 \\
\mathrm{gl} 2\end{array}$ & ,622 \\
\hline $\begin{array}{c}\text { No se aplican para } \\
\text { todas las especialidades } \\
\text { médicas, }\end{array}$ & $\begin{array}{c}17 \\
33 \%\end{array}$ & $42 \%$ & $\begin{array}{c}21 \\
48 \%\end{array}$ & $\begin{array}{c}23 \\
52 \%\end{array}$ & $\begin{array}{c}4 \\
80 \%\end{array}$ & $\begin{array}{c}1 \\
20 \%\end{array}$ & $\begin{array}{c}5,414 \\
\text { gl } 2\end{array}$ & 0,067 \\
\hline
\end{tabular}

La variable APLICACIÓN (Tabla 7), analizada a partir de siete preguntas, lo más resaltante es el $15 \%$ y $20 \%$ de me y mg que nunca han prescrito una FM en contraste con el $10 \%$ y $20 \%$ de m.e. y od. que prescriben a menudo. En el orden de me, mg y od prescriben muy poco y a veces el $75 \%, 35 \%$ y $80 \%$; el $67 \%, 50 \%$ y $60 \%$ indican que los pacientes nunca solicitan FM y el 31\%, 48\% y 20 que muy poco y a veces solicitan. Un resultado positivo constituye la ausencia de complicaciones al aplicar FM según indican el $60 \%, 37 \%$ y $40 \%$ de los médicos, y en el lado negativo $40 \%$, 63\% y $20 \%$ hay complicaciones. En cuanto a la identificación de necesidad de FM en problemas de salud de los pacientes de estos tres tipos de médicos, los odontólogos indican que se dan a veces en un $80 \%$, las opciones a veces y a menudo se dan el $59 \%$ y el $55 \%$ según los me y mg.

Respecto a la preferencia de las diferentes presentaciones de FM en términos generales y en el orden de ninguna preferencia, baja preferencia, media preferencia y alta preferencia, se tiene en: soluciones y suspensiones magistrales el 30\%, 22\%, $27 \%$ y el $21 \%$ respectivamente; en cremas, pomadas, pastas y geles magistrales: $16 \%, 29 \%$, 30\% y el $25 \%$; en capsula y polvos magistrales: $36 \%, 26 \%, 22 \%$ y $16 \%$.

Tabla 7: Resumen de las tablas de contingencias de la variable APLICACION sobre Formulación magistral y tipo de médico.

\begin{tabular}{|c|c|c|c|c|c|c|c|c|}
\hline \multirow{3}{*}{$\begin{array}{c}\text { Escala de } \\
\text { Likert }\end{array}$} & \multicolumn{7}{|c|}{ Sección: Aplicación } & \multirow{3}{*}{$\begin{array}{l}\text { Sign. Asint } \\
\text { Bilateral }\end{array}$} \\
\hline & \multicolumn{2}{|c|}{ Médicos especialistas } & \multicolumn{2}{|c|}{ Médicos generales } & \multicolumn{2}{|c|}{ Odontólogos } & \multirow{2}{*}{$\begin{array}{c}\text { Chi }^{2} \\
\text { Calc. }\end{array}$} & \\
\hline & Frecuencia & $\%$ & Frecuencia & $\%$ & Frecuencia & $\%$ & & \\
\hline \multicolumn{9}{|c|}{ ¿Ha prescrito formulación magistral a sus pacientes? } \\
\hline Nunca & 8 & $15 \%$ & 9 & $20 \%$ & 0 & $0 \%$ & \multirow{4}{*}{$\begin{array}{c}7,455 \\
\text { gl } 6\end{array}$} & \multirow[t]{4}{*}{,281 } \\
\hline Muy poco & 28 & $54 \%$ & 28 & $64 \%$ & 3 & $60 \%$ & & \\
\hline A veces & 11 & $21 \%$ & 7 & $16 \%$ & 1 & $20 \%$ & & \\
\hline A menudo & 5 & $10 \%$ & 0 & $0 \%$ & 1 & $20 \%$ & & \\
\hline \multicolumn{9}{|c|}{ ¿Sus pacientes solicitan formulación magistral? } \\
\hline Nunca & 35 & $67 \%$ & 22 & $50 \%$ & 3 & $60 \%$ & \multirow{4}{*}{$\begin{array}{c}8,856 \\
\text { gl } 6\end{array}$} & \multirow[t]{4}{*}{, 182} \\
\hline Muy poco & 11 & $21 \%$ & 15 & $34 \%$ & 1 & $20 \%$ & & \\
\hline A veces & 5 & $10 \%$ & 6 & $14 \%$ & 0 & $0 \%$ & & \\
\hline A menudo & 1 & $2 \%$ & 1 & $2 \%$ & 1 & $20 \%$ & & \\
\hline \multicolumn{9}{|c|}{ ¿La aplicación de fórmulas magistrales ha tenido complicaciones? } \\
\hline Nunca & 31 & $60 \%$ & 16 & $37 \%$ & 2 & $40 \%$ & \multirow{4}{*}{$\begin{array}{c}5,823 \\
\text { gl } 6\end{array}$} & \multirow[t]{4}{*}{,443 } \\
\hline Muy poco & 18 & $34 \%$ & 21 & $47 \%$ & 2 & $40 \%$ & & \\
\hline A veces & 2 & $4 \%$ & 6 & $13 \%$ & 1 & $20 \%$ & & \\
\hline A menudo & 1 & $2 \%$ & 1 & $3 \%$ & 0 & $0 \%$ & & \\
\hline \multicolumn{9}{|c|}{ ¿Existen problemas de salud que requieren de fórmulas magistrales? } \\
\hline Nunca & 3 & $6 \%$ & 3 & $7 \%$ & 0 & $0 \%$ & \multirow{4}{*}{$\begin{array}{c}4,801 \\
\mathrm{gl} 6\end{array}$} & \multirow[t]{4}{*}{, 570} \\
\hline Muy poco & 18 & $35 \%$ & 17 & $39 \%$ & 1 & $20 \%$ & & \\
\hline A veces & 21 & $40 \%$ & 14 & $32 \%$ & 4 & $80 \%$ & & \\
\hline A menudo & 10 & $19 \%$ & 10 & $23 \%$ & 0 & $0 \%$ & & \\
\hline
\end{tabular}




\begin{tabular}{|c|c|c|c|c|c|c|c|c|}
\hline Ninguna & 15 & $29 \%$ & 12 & $27 \%$ & 4 & $80 \%$ & \multirow{4}{*}{$\begin{array}{c}8,770 \\
\text { gl } 6\end{array}$} & \multirow[t]{4}{*}{, 187} \\
\hline Muy poca & 12 & $23 \%$ & 10 & $23 \%$ & 0 & $0 \%$ & & \\
\hline A veces & 12 & $23 \%$ & 15 & $34 \%$ & 0 & $0 \%$ & & \\
\hline A menudo & 13 & $25 \%$ & 7 & $16 \%$ & 1 & $20 \%$ & & \\
\hline \multicolumn{9}{|c|}{ sería su preferencia si prescribe o prescribiría cremas, pomadas, pastas y geles magistrales? } \\
\hline Ninguna & 10 & $19 \%$ & 5 & $12 \%$ & 1 & $20 \%$ & \multirow{4}{*}{$\begin{array}{c}7,652 \\
\text { gl } 6\end{array}$} & \multirow[t]{4}{*}{,265 } \\
\hline Muy poca & 11 & $21 \%$ & 16 & $36 \%$ & 2 & $40 \%$ & & \\
\hline A veces & 13 & $25 \%$ & 16 & $36 \%$ & 1 & $20 \%$ & & \\
\hline A menudo & 18 & $35 \%$ & 7 & $16 \%$ & 1 & $20 \%$ & & \\
\hline \multicolumn{8}{|c|}{ sería su preferencia si prescribe o prescribiría cápsulas y polvos magistrales? } & \\
\hline Ninguna & 23 & $44 \%$ & 13 & $29 \%$ & 1 & $20 \%$ & 7,623 & \multirow[t]{4}{*}{,267 } \\
\hline Muy poca & 11 & $21 \%$ & 15 & $34 \%$ & 0 & $0 \%$ & gl 6 & \\
\hline A veces & 10 & $19 \%$ & 10 & $23 \%$ & 2 & $40 \%$ & & \\
\hline A menudo & 8 & $16 \%$ & 6 & $14 \%$ & 2 & $40 \%$ & & \\
\hline
\end{tabular}

Fuente: H-IESS, HUA-UNACH, y HPAVR

Realizado por: Grupo de Investigación

Las pruebas de $\mathrm{Chi}^{2}$ en cada ítem de la variable APLICACIÓN a un nivel de confianza $\alpha=0,05$ indica no asociación.

Tabla 8: Resumen de las tablas de contingencias de la variable INTENCIÓN sobre Formulación magistral y tipo de médico.

\begin{tabular}{|c|c|c|c|c|c|c|c|c|}
\hline \multirow{3}{*}{$\begin{array}{c}\text { Escala de } \\
\text { Likert }\end{array}$} & \multicolumn{7}{|c|}{ Sección: Intención } & \multirow{3}{*}{$\begin{array}{l}\text { Signf. Asint. } \\
\text { Bilateral }\end{array}$} \\
\hline & \multicolumn{2}{|c|}{ Médicos especialistas } & \multicolumn{2}{|c|}{ Médicos generales } & \multicolumn{2}{|c|}{ Odontólogos } & \multirow{2}{*}{$\begin{array}{l}\text { Chi }^{2} \\
\text { Calc. }\end{array}$} & \\
\hline & recuencia & $\%$ & Frecuencia & $\%$ & Frecuencia & $\%$ & & \\
\hline \multicolumn{9}{|c|}{ ¿Tiene interés en ampliar sus conocimientos sobre formulación magistral? } \\
\hline Muy en desacuerdo & 0 & $0 \%$ & 0 & $0 \%$ & 0 & $0 \%$ & \multirow{5}{*}{$\begin{array}{c}3,150 \\
\text { g1 } 8\end{array}$} & \multirow[t]{5}{*}{,790 } \\
\hline En desacuerdo & 1 & $2 \%$ & 1 & $2 \%$ & 0 & $0 \%$ & & \\
\hline Indeciso & 6 & $11 \%$ & 2 & $7 \%$ & 0 & $0 \%$ & & \\
\hline De acuerdo & 28 & $54 \%$ & 24 & $55 \%$ & 4 & $80 \%$ & & \\
\hline Muy de acuerdo & 17 & $33 \%$ & 17 & $36 \%$ & 1 & $20 \%$ & & \\
\hline \multicolumn{9}{|c|}{ ¿Le gustaría asistir a un programa de actualización sobre formulación magistral? } \\
\hline Muy en desacuerdo & 3 & $6 \%$ & 0 & $0 \%$ & 0 & $0 \%$ & \multirow{5}{*}{$\begin{array}{c}5,468 \\
\text { g1 } 8\end{array}$} & \multirow[t]{5}{*}{0,707} \\
\hline En desacuerdo & 2 & $4 \%$ & 1 & $2 \%$ & 0 & $0 \%$ & & \\
\hline Indeciso & 7 & $13 \%$ & 3 & $7 \%$ & 1 & $20 \%$ & & \\
\hline De acuerdo & 25 & $48 \%$ & 24 & $55 \%$ & 3 & $60 \%$ & & \\
\hline Muy de acuerdo & 15 & $29 \%$ & 16 & $36 \%$ & 1 & $20 \%$ & & \\
\hline \multicolumn{9}{|c|}{ ¿A través de qué medios le gustaría informarse y ampliar sus conocimientos sobre formulación magistral? } \\
\hline \multirow{3}{*}{ Radio } & NO & SI & NO & SI & NO & SI & & \multirow{3}{*}{,833 } \\
\hline & 50 & 2 & 43 & 1 & 5 & 0 & 0,366 & \\
\hline & $96 \%$ & $4 \%$ & $98 \%$ & $2 \%$ & $100 \%$ & $0 \%$ & $\mathrm{gl}=2$ & \\
\hline \multirow[t]{2}{*}{ TV } & 48 & 4 & 39 & 5 & 4 & 1 & \multirow[t]{2}{*}{0,962} & \multirow[t]{2}{*}{ 618 } \\
\hline & $92 \%$ & $8 \%$ & $89 \%$ & $11 \%$ & $80 \%$ & $20 \%$ & & \\
\hline \multirow[t]{2}{*}{ Internet } & 19 & 33 & 20 & 24 & 3 & 2 & \multirow[t]{2}{*}{1,514} & \multirow[t]{2}{*}{,469 } \\
\hline & $37 \%$ & $63 \%$ & $45 \%$ & $55 \%$ & $60 \%$ & $40 \%$ & & \\
\hline \multirow[t]{2}{*}{ Conferencia } & 16 & 36 & 13 & 31 & 3 & 2 & \multirow[t]{2}{*}{1,965} & \multirow[t]{2}{*}{, 374} \\
\hline & $31 \%$ & $69 \%$ & $29 \%$ & $71 \%$ & $60 \%$ & $40 \%$ & & \\
\hline \multirow[t]{2}{*}{ Folletos } & 30 & 22 & 25 & 19 & 3 & 2 & \multirow[t]{2}{*}{0,022} & \multirow[t]{2}{*}{989} \\
\hline & $58 \%$ & $42 \%$ & $57 \%$ & $43 \%$ & $60 \%$ & $40 \%$ & & \\
\hline
\end{tabular}

Fuente: H-IESS, HUA-UNACH, y HPAVR

Realizado por: Grupo de Investigación

Según los resultados de la variable INTENCION (tabla 8), en las opciones de acuerdo y muy de acuerdo en estar interesado en ampliar los conocimientos sobre FM se identifican el $87 \%, 94 \%$ y $100 \%$ de me, $\mathrm{mg}$, y od. Respecto a asistir a un programa de actualización sobre FM, están de acuerdo y muy de acuerdo el 77\%, 91\% y $80 \%$ de los médicos encuestados. En general manifiestan los médicos no les gustaría informarse y ampliar sus conocimientos sobre formulación magistral por medio de la radio y televisión, y si les agradaría por conferencias (68\%), internet (58\%) y folletos (43\%). Las pruebas de $\mathrm{Ch}^{2}$ en 
cada ítem de esta variable a un nivel de confianza $\alpha=0,05$ indica no asociación entre las variables, es decir se acepta la hipótesis nula.

\section{- De la encuesta a Bioquímicos Farmacéuticos}

De las encuestas aplicadas a Bioquímicos farmacéuticos (BQFs) que trabajan en Riobamba en los hospitales H-IESS, HUA-UNACH, y HPAVR, en la Botica Bristol (BB), y en la Farmacia RioPharma (FR), son graduados en la ESPOCH (95\%), Universidad de Cuenca (5\%) y Universidad de Guayaquil (5\%), se presentan los siguientes resultados:

Tabla 9: Frecuencias y porcentajes de la variable CONOCIMIENTOS sobre Formulación magistral de los Bioquímicos Farmacéuticos.

\begin{tabular}{|c|c|c|}
\hline \multicolumn{3}{|c|}{ Sección: Conocimientos } \\
\hline \multicolumn{3}{|c|}{ ¿Cuánto considera que conoce sobre formulación magistral? } \\
\hline Escala de Likert & Frecuencia & Porcentaje \\
\hline Insuficiente & 2 & $10 \%$ \\
\hline Regular & 5 & $25 \%$ \\
\hline Aceptable & 12 & $60 \%$ \\
\hline Excelente & 1 & $5 \%$ \\
\hline \multicolumn{3}{|c|}{ ¿Cuánta importancia se dio a la formulación magistral en su formación de pregrado? } \\
\hline Insuficiente & 5 & $15 \%$ \\
\hline Regular & 9 & $45 \%$ \\
\hline Aceptable & 5 & $25 \%$ \\
\hline Excelente & 3 & $15 \%$ \\
\hline \multicolumn{3}{|c|}{ ¿Cuánto conoce sobre la legislación de fórmulas magistrales? } \\
\hline Insuficiente & 4 & $20 \%$ \\
\hline Regular & 11 & $55 \%$ \\
\hline Aceptable & 5 & $25 \%$ \\
\hline Excelente & 0 & $0 \%$ \\
\hline \multicolumn{3}{|c|}{ ¿Cuánto conoce sobre algún formulario nacional? } \\
\hline Insuficiente & 5 & $25 \%$ \\
\hline Regular & 10 & $50 \%$ \\
\hline Aceptable & 2 & $10 \%$ \\
\hline Excelente & 3 & $15 \%$ \\
\hline \multicolumn{3}{|c|}{ ¿Cuál es su conocimiento sobre las buenas prácticas de elaboración de fórmulas magistrales? } \\
\hline Insuficiente & 1 & $5 \%$ \\
\hline Regular & 6 & $30 \%$ \\
\hline Aceptable & 10 & $50 \%$ \\
\hline Excelente & 3 & $15 \%$ \\
\hline \multicolumn{3}{|c|}{ ¿Cuál es su conocimiento sobre los requisitos de calidad para fórmulas magistrales? } \\
\hline Insuficiente & 2 & $10 \%$ \\
\hline Regular & 5 & $25 \%$ \\
\hline Aceptable & 12 & $60 \%$ \\
\hline Excelente & 1 & $5 \%$ \\
\hline
\end{tabular}

Fuente: H-IESS, HUA-UNACH, HPAVR, BB y FR

Elaborado por: Grupo de Investigación

Sobre los conocimientos de FM los BQFs señalan un 60\% en aceptable. En la importancia que le dieron a las FM en sus estudios el $45 \%$ indica regular, aceptable el $25 \%$ y excelente $15 \%$, sobre la legislación indican regular el 55\% y, 25\% aceptable; En cuanto al conocimiento de algún formulario nacional el 50\% indica regular, aceptable $10 \%$ y excelente $15 \%$. Sobre buenas prácticas de elaboración y requisitos de calidad de las FM el $50 \%$ y $60 \%$ se halla en la escala aceptable, $35 \%$ entre insuficiente y regular, y de excelente el $15 \%$ y $5 \%$ (tabla 9 ).

Respecto a la variable OPINION (tabla 10), en cuanto a considerar importante el uso de FM: de acuerdo el 35\%, muy de acuerdo el 65\%. El 85\% están de acuerdo y muy de 
acuerdo en que se requieren FM en sus trabajos. En desacuerdo y muy en desacuerdo el $30 \%$ y el $60 \%$ respectivamente en considerar obsoleto el uso de fórmulas magistrales, y un $10 \%$ está muy de acuerdo. En cuanto a estar preparados para elaborar FM, entre indecisos y en desacuerdo suman el $20 \%$, el $60 \%$ y $20 \%$ están de acuerdo y muy de acuerdo. Opinan estar de acuerdo el 30\%, y muy de acuerdo el $70 \%$ en que la elaboración de FM amplía sus oportunidades de empleo.

Tabla 10: Frecuencias y porcentajes de la variable OPINIÓN sobre Formulación magistral de los Bioquímicos Farmacéuticos.

\begin{tabular}{|c|c|c|}
\hline \multicolumn{3}{|c|}{ Sección: Opinión } \\
\hline ¿Considera importante el uso & & \\
\hline Escala de Likert & Frecuencia & Porcentaje \\
\hline Muy en desacuerdo & 0 & $0 \%$ \\
\hline En desacuerdo & 0 & $0 \%$ \\
\hline Indeciso & 0 & $0 \%$ \\
\hline De acuerdo & 7 & $35 \%$ \\
\hline Muy de acuerdo & 13 & $65 \%$ \\
\hline \multicolumn{3}{|c|}{ ¿En su establecimiento de trabajo requieren de fórmulas magistrales? } \\
\hline Muy en desacuerdo & 0 & $0 \%$ \\
\hline En desacuerdo & 1 & $5 \%$ \\
\hline Indeciso & 2 & $10 \%$ \\
\hline De acuerdo & 11 & $55 \%$ \\
\hline Muy de acuerdo & 6 & $30 \%$ \\
\hline \multicolumn{3}{|c|}{ ¿Considera anticuado el uso de formulaciones magistrales? } \\
\hline Muy en desacuerdo & 12 & $60 \%$ \\
\hline En desacuerdo & 6 & $30 \%$ \\
\hline Indeciso & 0 & $0 \%$ \\
\hline De acuerdo & 0 & $0 \%$ \\
\hline Muy de acuerdo & 2 & $10 \%$ \\
\hline \multicolumn{3}{|c|}{ ¿Está preparado para elaborar fórmulas magistrales demandadas actualmente? } \\
\hline Muy en desacuerdo & 0 & $0 \%$ \\
\hline En desacuerdo & 1 & $5 \%$ \\
\hline Indeciso & 3 & $15 \%$ \\
\hline De acuerdo & 12 & $60 \%$ \\
\hline Muy de acuerdo & 4 & $20 \%$ \\
\hline \multicolumn{3}{|c|}{ ¿La elaboración de fórmulas magistrales amplía sus oportunidades de empleo? } \\
\hline Muy en desacuerdo & 0 & $0 \%$ \\
\hline En desacuerdo & 0 & $0 \%$ \\
\hline Indeciso & 0 & $0 \%$ \\
\hline De acuerdo & 6 & $30 \%$ \\
\hline Muy de acuerdo & 14 & $70 \%$ \\
\hline
\end{tabular}

Fuente: H-IESS, HUA-UNACH, HPAVR, BB y FR

Elaborado por: Grupo de Investigación

Sobre la APLICACION de FM (tabla 11), el 15\% de los BQFs indican haberlas elaborado a menudo, muy poco y a veces el $70 \%$, y nunca el $15 \%$. El $55 \%$ señala que nunca la aplicación de FM ha presentado complicaciones y el $45 \%$ muy poco y a veces. El $60 \%$ de los pacientes nunca solicitan FM. En cuanto a elaborar las diferentes presentaciones de FM, señalan una frecuencia del 55\% de nunca en soluciones y suspensiones, $35 \%$ en cremas, pomadas, pastas y geles magistrales, y en capsulas y polvos, entre $10 \%$ al $15 \%$ prepararían a menudo cualquiera de las presentaciones.

Tabla 11: Frecuencias y porcentajes de la variable APLICACIÓN sobre Formulación magistral de los Bioquímicos Farmacéuticos.

\begin{tabular}{ccc}
\hline \multicolumn{3}{c}{ Sección: Aplicación } \\
\hline ¿Ha elaborado formulación magistral en su ejercicio profesional? & Precuencia & $15 \%$ \\
Escala de Likert & 3 & $45 \%$ \\
Nunca & 9 & $25 \%$ \\
Muy poco & 5 & \\
A veces & 5
\end{tabular}




\begin{tabular}{|c|c|c|}
\hline A menudo & 3 & $15 \%$ \\
\hline \multicolumn{3}{|c|}{ ¿Los pacientes solicitan formulación magistral? } \\
\hline Nunca & 12 & $60 \%$ \\
\hline Muy poco & 7 & $35 \%$ \\
\hline A veces & 1 & $5 \%$ \\
\hline A menudo & 0 & $0 \%$ \\
\hline \multicolumn{3}{|c|}{ ¿En caso de haber elaborado fórmulas magistrales se han presentado complicaciones? } \\
\hline Nunca & 11 & $55 \%$ \\
\hline Muy poco & 6 & $30 \%$ \\
\hline A veces & 3 & $15 \%$ \\
\hline A menudo & 0 & $0 \%$ \\
\hline \multicolumn{3}{|c|}{ ¿Existen problemas de salud que requieren fórmulas magistrales? } \\
\hline Nunca & 0 & $0 \%$ \\
\hline Muy poco & 4 & $20 \%$ \\
\hline A veces & 14 & $70 \%$ \\
\hline A menudo & 2 & $10 \%$ \\
\hline \multicolumn{3}{|c|}{ ¿Cuál sería su frecuencia si elabora o elaboraría soluciones y suspensiones magistrales? } \\
\hline Nunca & 11 & $55 \%$ \\
\hline Muy poco & 3 & $15 \%$ \\
\hline A veces & 4 & $20 \%$ \\
\hline A menudo & 2 & $10 \%$ \\
\hline \multicolumn{3}{|c|}{ ¿Cuál sería su frecuencia si elabora o elaboraría cremas, pomadas, pastas y geles magistrales? } \\
\hline Nunca & 7 & $35 \%$ \\
\hline Muy poco & 8 & $40 \%$ \\
\hline A veces & 3 & $15 \%$ \\
\hline A menudo & 2 & $10 \%$ \\
\hline \multicolumn{3}{|c|}{ ¿Cuál sería su frecuencia si elabora o elaboraría cápsulas y polvos magistrales? } \\
\hline Nunca & 7 & $35 \%$ \\
\hline Muy poco & 6 & $30 \%$ \\
\hline A veces & 4 & $20 \%$ \\
\hline A menudo & 3 & $15 \%$ \\
\hline
\end{tabular}

Fuente: H-IESS, HUA-UNACH, HPAVR, BB y FR

Elaborado por: Grupo de Investigación

A continuación, se presentan los resultados sobre la variable INTENCION (Tabla 12), en la que los profesionales BQFs muestran interés en ampliar sus conocimientos sobre FM, el $30 \%$ y el $70 \%$ están de acuerdo y muy de acuerdo en este punto. En cuanto a la asistencia a programas de actualización solo un 5\% se manifiesta indeciso, el $25 \%$ está de acuerdo y el $70 \%$ muy de acuerdo. Sobre los medios a través de los cuales les gustaría adquirir esta actualización no hay una marcada preferencia sobre internet, conferencias y folletos.

Tabla 12: Frecuencias y porcentajes de la variable INTENCIÓN sobre Formulación magistral de los Bioquímicos Farmacéuticos.

\begin{tabular}{ccc}
\hline & Sección: Intención & \\
\hline ¿Tiene interés en ampliar sus conocimientos sobre formulación magistral? & Porcentaje \\
Escala de Likert & Frecuencia & $0 \%$ \\
Muy en desacuerdo & 0 & $0 \%$ \\
En desacuerdo & 0 & $0 \%$ \\
Indeciso & 0 & $30 \%$ \\
De acuerdo & 6 & $70 \%$ \\
Muy de acuerdo & 14 & $0 \%$ \\
¿Luy en desacuerdo & $0 \%$ \\
En desacuerdo & 0 & $5 \%$ \\
Indeciso & 0 & $25 \%$ \\
De acuerdo & 1 & $70 \%$ \\
Muy de acuerdo & 14 & \\
¿A través de qué medios le gustaría informarse y ampliar sus conocimientos sobre formulación & \\
magistral? asistir a un programa de actualización sobre formulación magistral? & \\
TV & 2 & $29 \%$ \\
Internet & 6 & $39 \%$ \\
Folletos & 8 & $23 \%$ \\
\hline
\end{tabular}

Fuente: H-IESS, HUA-UNACH, HPAVR, BB y FR

Elaborado por: Grupo de Investigación 
- $\quad$ Análisis de escala Likert

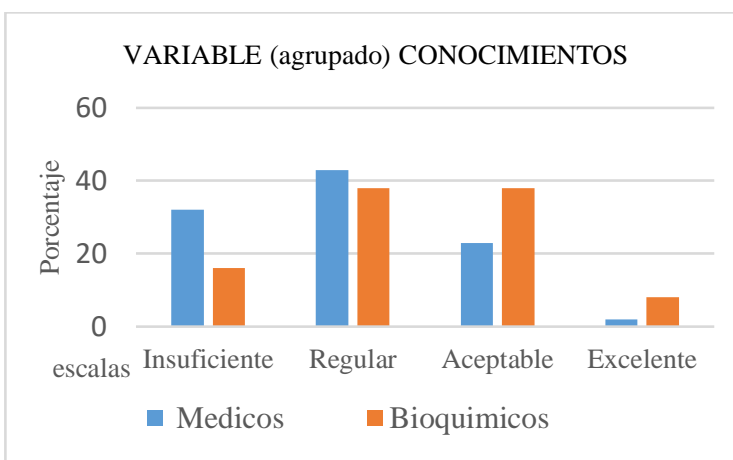

Gráfico 1A: Variable Conocimientos

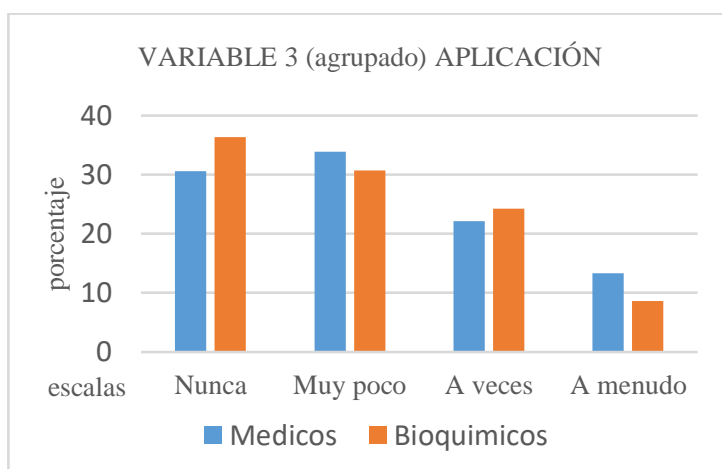

Gráfico 1C: Variable (agrupada) Aplicación

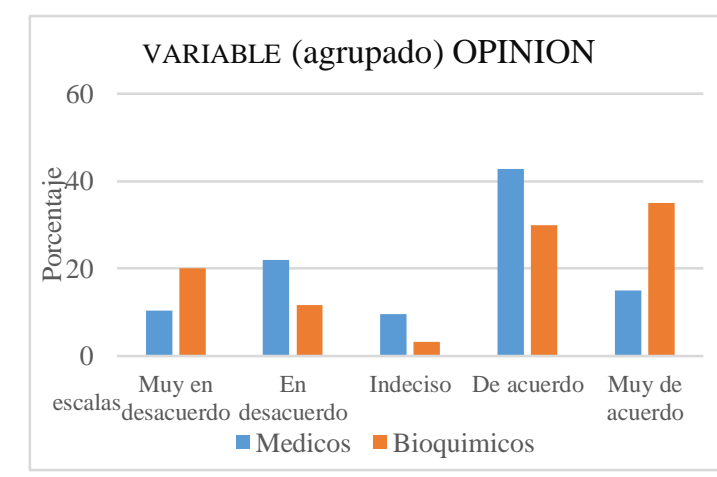

Gráfico 1B: Variable Opinión

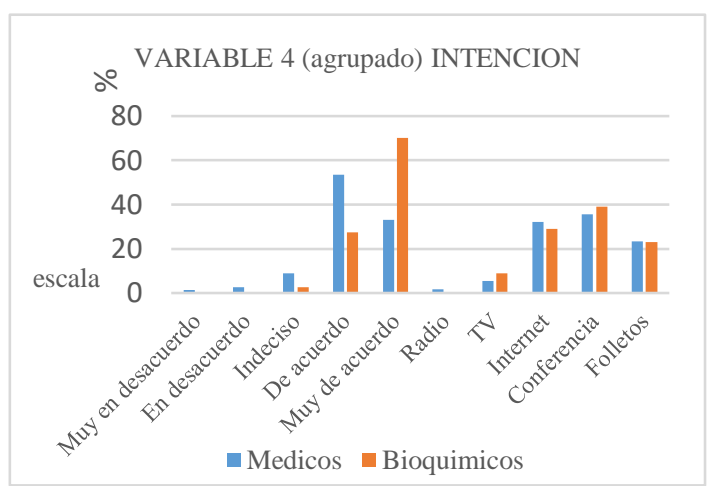

Gráfico 1D: Variable (agrupada) Opinión

Gráfico 1: Representación gráfica de las variables agrupadas

Fuente: Resultados de encuestas dirigidas a médicos y BQF del H-IESS, HUA-UNACH, HPAVR, BB y FR Elaborado por: Grupo de Investigación

Los resultados de las pruebas $\mathrm{Chi}^{2}$ entre la variable tipo de médico y las variables: conocimiento, opinión, aplicación e intención; y sus subvariables, a un nivel de confianza $\alpha=0,05$ indican no asociación entre dichas variables, es decir se acepta la hipótesis nula.

Ho: Las variables conocimientos, opinión, aplicación, e intención sobre la formulación magistral son independientes de la variable tipo de médico.

Al no haber estadísticamente una diferenciación por tipo de médicos, se los agrupa, y se realiza un análisis de escala de Likert de BQFs y médicos, facilitando a través de la agrupación de datos la apreciación de las diferencias entre los prescriptores y elaboradores de fórmulas magistrales.

Los médicos en cuanto a la variable conocimientos sobre las FM presentan el 75\% entre las escalas insuficiente y regular, en contraste con los BQFs con 54\%. En las escalas aceptable y excelente se invierten los resultados, 25\% para los médicos y $46 \%$ los BQFs.

En la gráfica 1B, respecto a opinión sobre las características positivas de las FM los médicos están en desacuerdo, y en los calificativos negativos (ej anticuado, obsoleto) opinan estar más de acuerdo, los BQFs opinan a la inversa, otra diferencia marcada es el $80 \%$ de BQFs que están de acuerdo y muy de acuerdo en estar preparados para elaborar 
FM, mientras el $43 \%$ de los médicos están de acuerdo y muy de acuerdo que no hay profesionales que elaboren FM.

Respecto a la variable aplicación (grafico 1C), en cuanto a haber elaborado (BQFs) o haber prescrito (médicos) FM, si las solicitan los pacientes, si han presentado complicaciones su aplicación; los más altos porcentajes están en las escalas nunca y muy poco, y en porcentajes más bajos las opciones a veces y a menudo, estos resultados se dan de manera similar en los dos grupos.

Sobre el interés en ampliar conocimientos y asistir a un programa de actualización de FM, manifiestan mayor porcentaje y entusiasmo los BQF anotando un alto porcentaje en muy de acuerdo y de acuerdo, el mayor porcentaje de los médicos está en la escala de acuerdo y en menor porcentaje en muy de acuerdo. De mayor a menor preferencia de medios para actualizar sus conocimientos, los dos grupos indican: conferencias, internet y folletos.

\section{Discusión}

La variable agrupada conocimientos sobre FM evidencia mayoritariamente un nivel insuficiente e irregular del 75\% de los médicos, y 54\% de los BQFs. Los médicos también han manifestado en entrevistas que en sus mallas curriculares de estudio no incluyen la formulación magistral, por lo tanto, su conocimiento se crea en el ejercicio de su profesión, y de la transferencia entre galenos, la tendencia es aminorar los médicos con el conocimiento para prescribir correctamente formulaciones magistrales, por lo tanto, esta vía de aprendizaje para las nuevas generaciones de médicos va desapareciendo. Siguiendo con el análisis agrupado el $46 \%$ de los BQFs presentan la variable conocimiento en las escalas aceptable y excelente, esto puede deberse a su propio interés o a la importancia que dio la universidad, o por la falta de rentabilidad de algunas fórmulas para cuya elaboración se necesitan ingredientes difíciles de conseguir, sumado a la muy poca prescripción por parte de los médicos, incidiendo en los costos. Médicos y BQFs consideran en un alto porcentaje importante el uso de FM. En el presente la industria farmacéutica cuenta con una gran cantidad de fármacos, sin embargo, las FM siguen teniendo una gran importancia en el tratamiento, especialmente para ciertos grupos poblacionales como son los pacientes dermatológicos y pediátricos, creando una disposición a la personalización de la medicación (Baixauli, 2015). La medicación personalizada requiere de la cooperación y comunicación eficiente entre el farmacéutico y el médico, especialmente para dar solución a posibles problemas que se presenten (Fernández \& Bustamante, 2020).

Los BQFs tienen conocimientos aceptables $(50 \%)$ y regular $(30 \%)$ sobre las buenas prácticas de elaboración para FM. Según la OMS la elaboración de FM debe cumplir con controles estandarizados que garanticen al consumidor la calidad de estos medicamentos. Las buenas prácticas de elaboración de medicamentos son guías que se utilizan tanto para preparados magistrales y oficinales, y se pueden adecuar para el caso de medicamentos personalizados, de manera que el dominio de estas herramientas es imprescindible para los farmacéuticos, quienes además contribuyen con la vigilancia que es crucial para 
proteger a los pacientes (OMS, 2018), también con el control de calidad de medicamentos en su sitio de trabajo, lo que les demanda conocer los requisitos de calidad para FM, en este ítem el $60 \%$ responde tener conocimientos aceptables. Para la elaboración de FM se aplican controles de calidad para todos y cada uno de los procesos que requiera una formulación, asegurando que el medicamento cumpla con las especificaciones reguladas, así por ejemplo en el proceso de pesaje y medición cada paso es controlado (Ramos, Herreros, \& de Rosales, 2020; ARSA, 2020)

En cuanto a la variable opinión, el 91,3\% de los médicos y el 100\% de BQF están de acuerdo y muy de acuerdo en considerar importante el uso de FM. Respecto a las ventajas el 45,29\% de los médicos no consideran como ventajas la personalización de los tratamientos, los bajos costos, la flexibilidad de dosis y el hecho de que las FM. cubren el desabastecimiento de medicamentos, mientras que para el $54,7 \%$ si son ventajas. La FM permite personalizar un medicamento que contribuye con una real solución para tratar un sin número de pacientes del entorno asistencial, permite dosificaciones más flexibles según necesidades de cada paciente siempre logrando la posología correcta. Los farmacéuticos del hoy y el mañana pueden ampliar y desarrollar sus capacidades y oportunidades en torno al servicio de formular (Baixauli, 2015; Peña, 2020).

Sobre las desventajas de la FM: falta de locales de elaboración, medicamentos obsoletos, no poseen registro sanitario, no son aplicables para todas las especialidades, los médicos opinan: en un $47 \%$ no las consideran desventajas y el $53 \%$ si las consideran desventajas. La falta de lugares de elaboración de FM es evidente, si esta labor está disminuyendo, evidentemente los locales y farmacéuticos encargados también. Ciertamente las FM no se aplican a todas las especialidades médicas, lo confirma Morales, et al., (2008) en investigaciones que encuentran aplicables estos medicamentos en las terapias hospitalarias de diversas especialidades médicas, se citan la dermatología, pediatría y oftalmología.

En las escalas de acuerdo y muy de acuerdo: el $85 \%$ de los BQFs indican que se requieren FM en sus trabajos, esto amplia el área de trabajo para los $\mathrm{BQF}$, considerando que este servicio en mínimo en Riobamba, especialmente en el sistema de salud principalmente hospitales. En las farmacias la FM brinda un importante servicio en la resolución de problemas que se dan en el día a día de la práctica clínica (Gonzales, et al., 2016; Rodriguez, et al., 2017); el 80\% consideran estar preparados para elaborar las FM demandadas actualmente, opinión que corresponde a la formación académica amplia, actualizada, y enfocada en la aplicación íntegra de diversos tratamientos terapéuticos que brindan los BQFs (Jacobi, 2016). En contraste el $43 \%$ de los médicos opinan que no hay profesionales que elaboren FM, esta baja en la elaboración de estos medicamentos coincide con estudios (Castillo, 2004) que señalan además como causantes de esta tendencia al desinterés y abandono de esta práctica por parte de los BQFs..

El 100\% de los BQF señala que tienen mayor oportunidad de trabajo, los BQF formulistas contribuyen a la individualización y acceso a medicamentos, elaboran diferentes formas de dosificación o las combinan para dosis o concentraciones que no se hallan usualmente 
(PwC, 2011). El 90\% está en desacuerdo y muy en desacuerdo en considerar obsoleto el uso de FM. En el área de la aplicación de las FM enfocado en la elaboración para los BQFs, y en la prescripción para los médicos, indican muy poco el $45 \%$ y el $48 \%$ respectivamente. Los pocos galenos que prescriben a veces o a menudo generalmente son los dermatólogos, y odontólogos, para quienes la FM es útil, para la mayoría de los prescriptores no lo es, estas respuestas evidencian que no conocen el uso de FM para ciertas enfermedades. Respecto a si los pacientes solicitan FM también es similar en la escala nunca con el $60 \%$ en BQF y $59 \%$ en médicos. Con las preguntas ¿ha presentado complicaciones la aplicación de FM? los resultados son similares, los más altos porcentajes están en las escalas nunca y muy poco, y en porcentajes más bajos las opciones a veces y a menudo.

Respecto a la cual sería la frecuencia de elaboración de soluciones y suspensiones; de pomadas, cremas, pastas y geles, y, de capsulas y polvos, los BQF responden nunca el $55 \%, 35 \%$ y $35 \%$, y muy poco $15 \%, 40 \%$ y $30 \%$ respectivamente, en menor frecuencia las escalas a veces y a menudo $30 \%, 25 \%$ y $35 \%$, en general se visualizan elaborando pocas FM en cualquiera de las presentaciones. Respecto a los prescriptores, los odontólogos indican un $80 \%$ de ninguna preferencia en prescribir soluciones y suspensiones, sin embargo, en Oftalmología se emplean soluciones para los casos diagnosticados con glaucoma. La prescripción de cremas, pomadas, pastas y geles magistrales, fue altamente preferida por $\mathrm{mg}$, estas presentaciones son demandadas en Dermatología para enfermedades de la piel, en Pediatría, por ejemplo, en casos de pañalitis. Los me anotaron el $44 \%$ en ninguna preferencia respecto a capsulas y polvos magistrales, el $34 \%$ de los mg baja preferencia, $40 \%$ de los odontólogos selecciono media preferencia; la especialidad de Ginecología y Odontología disponen de formulaciones que son de fácil mezclado y envasado, con estabilidad química y diferentes usos. Nuevamente se evidencia en los grupos de médicos encuestados que no conocen de la variedad de FM, falta información y y que el BQF brinde formulaciones para las diferentes especialidades. Las presentaciones mayormente preferidas son las capsulas y polvos magistrales, se elaboran con facilidad, se dispone de las materias primas, tienen excelente demanda comercial y menores costos, y permiten un mejor margen de beneficio su manufactura.

Tanto BQFs como médicos manifiestan interés en ampliar sus conocimientos y asistir a programas de actualización de FM, anotando mayor porcentaje los BQFs, de forma similar los dos grupos tienen de mayor a menor preferencia de medios para actualizar sus conocimientos: conferencias, internet y folletos. La predisposición a actualizar sus conocimientos e innovarse, principalmente favorece a los pacientes, a quienes se ofrecería una solución farmacoterapéutica más factible; también a los médicos, quienes tendrían un aumento en sus alternativas terapéuticas; a los BQF formulistas, quienes estimularían su rol de formular y ampliarían su área de trabajo, y al Estado y sus programas de salud, que podrían agregar otras opciones, con seguridad, calidad y bajos costos, en beneficio de los ciudadanos, ampliando el acceso a medicamente y por lo tanto mejorando la atención a la salud (Abarca \& Marro, 2016). La medicina está cambiando en los últimos años y la farmacia está cambiando con ella, ha pasado de una farmacia tradicional que casi 
exclusivamente tendía a "tratar la enfermedad" a una farmacia moderna que da cada vez da más importancia a la "prevención en la salud" acorde a los retos que se vayan presentando, como la actual situación de pandemia, de manera que el farmacéutico tiene diversos y amplios campos de salud que atender (FIP, 2020).

\section{Conclusiones.}

- Las variables: conocimientos, opinión, aplicación, e intención sobre la formulación magistral estadísticamente son independientes de la variable tipo de médico. Los médicos presentan un insuficiente y regular conocimiento sobre diferentes aspectos de la formulación magistral, también han manifestado no contar en su malla curricular con estos conocimientos, en consecuencia, los médicos no están preparados para prescribir estos medicamentos y considerarlos como una opción apropiada en especialidades, esta baja prescripción incide en la demanda de elaboración. Los BQFs si bien tienen un mejor conocimiento de las fórmulas magistrales respecto a los médicos, no reportan una amplia diferencia como es de esperarse dada su especialidad, en general un $46 \%$ están en las escalas aceptable y excelente en conocimiento, en contraste el $80 \%$ opinan que están preparados para elaborar FM y el 100\% las considera una oportunidad de trabajo, mientras el $43 \%$ de los médicos opinan que no hay profesionales que elaboren FM, se identifica un vacío por parte de los BQFs, se requiere difundir y resaltar la amplia gama de formulaciones que existen para las diferentes necesidades médicas y que estas cumplen con los estándares de calidad de los medicamentos industriales ya que los médicos las consideran obsoletas y sin registro sanitario, el alto interés de todos los encuestados en ampliar conocimientos y asistir a programas de actualización de FM facilita a la academia diseñar y ofrecer estos programas; es necesario atender al sector más importante, los pacientes, ya que el $85 \%$ de los pacientes nunca y muy poco solicitan formulaciones. Existe una desarticulación, no existe colaboración y comunicación entre farmacéuticos y el Ministerio de Salud Pública, entre farmacéuticos-médico-paciente, que active una mayor prescripción, una eficiente y más amplia elaboración de FM, la acción y función principal de los farmacéuticos, que es orientar sus servicios a los clientes y al acceso a las ventajas que ofrece la formulación magistral.

\section{Referencias bibliográficas.}

Abarca, E., \& Marro, D. (2016). El desabastecimiento de los medicamentos: ¿qué hay detrás? Causas, consecuencias y una buena alternativa. ActasDermosifiliográficas, 107(3), 178-182. doi:10.1016/j.ad.2015.11.003

ARCSA. (5 de 3 de 2020). NTS Farmacias y Botiquines. Recuperado el 8 de 4 de 2021, de https://www.controlsanitario.gob.ec/wpcontent/uploads/downloads/2020/03/05.03.2020_NTS_farmacias-ybotiquines.pdf 
Bernal, C. (2016). Metodología de la Investigación: Administración, economía, humanidades y ciencias sociales ( $4^{\mathrm{a}}$ ed. ed.). Bogota, Colombia: Pearson.

Castillo, A. (2004). Estudio de la formulación magistral en oficina de farmacia desde 1985 a 2000 y su legislación correspondiente (Tesis) (Doctoral). (U. C. Madrid, Ed.) Recuperado el 8 de 4 de 2021

Com, S., Ackerman, S., \& Postolski, G. (2013). Metodologia de la Investigacion. Buenos Aires, Argentina: Ediciones del Aula Taller.

Corral, A. A. (2006). Formulación magistral, necesidad terapéutica en el siglo XXI. Rockville: Academia de Farmacia Santa María de España de la Región de Murcia.

Fernández, O. M., \& Bustamante, M. M. (2020). La creaciòn de polìticas pùblicas para la elaboraciòn de fòrmulas magistrales y preparados oficinales como derecho del paciente en el Ecuador. Guayaquil: Universidad de Guayaquil (UG).

FIP. (25 de 9 de 2020). PortalFarma. (F. I. Farmaceutica, Editor) Recuperado el 8 de 4 de 2021, de Día Mundial del Farmacéutico 2020 - 11. Transformando la salud global:

https://www.portalfarma.com/Profesionales/campanaspf/categorias/Paginas/Vari os/2020-dia-mundial-farmaceutico.aspx

Garcia, M., \& Molinero, M. (2014). Formulacion Magistral (1a ed ed.). Madrid, España: Ediciones Paraninfo.

Godínez, R. R., Aceves, P. P., \& Schifter, A. L. (13 de julio de 2019). La Industria Nacional Químico-Farmacéutica, S.A. de C.V. y P.E. (1949-1964). Un modelo sobresaliente de organización científica y empresarial. Educación química, 30(1), 149-160. doi:http://dx.doi.org/10.22201/fq.18708404e.2019.1.65578

Hernandez, S. R., Fernandez, C. C., \& Baptista, L. M. (2014). Metodología de la Investigación. ( $6^{\mathrm{a}}$ ed. ed.). (S. A. Interamericana Editores, Ed.) México: McGraw Hill.

Jacobi, J. (2016). Farmaceuticos clinicos profesionales esenciales del equipo de atencion clinica . Revista Medica Clinica La Condes, 27 (Issue 5), 578 - 584. doi:https://doi.org/10.1016/j.rmclc.2016.09.004

Lamberts, J. T., Vandiver, J. W., Daugherty, K. K., DiVall, M. V., Lebovitz, L., \& Roberts, S. C. (2021). Perceptions of programs that orient non-practice faculty to the pharmacy profession: A pilot study. Currents in Pharmacy Teaching and Learning, 3(4), e2021. doi:https://doi.org/10.1016/j.cptl.2021.03.004

Morales-Carpi, C., Chover, J. N., Lobatón, C. R., Estañ, L., \& Rubio, E. (2008). Medicamentos utilizados en pediatría extrahospitalaria: ¿disponemos de 
información suficiente? Anales de pediatria, 68(5), 439-446. doi: $10.1157 / 13120040$

OMS. (2018). Sistema Mundial de Vigilancia y Monitoreo de productos medicos de calidad subestandar y falsificados. doi:ISBN 978-92-4-351342-3

OPS/OMS. (2012). Salud en Sudamerica. Panorama de la situacion de salud y de las politicas y sistemas de salud. Washington DC. Recuperado el 23 de 03 de 2021, de

https://www.paho.org/chi/images/PDFs/salud\%20en\%20sam\%202012\%20(ene. 13).pdf

Ortiz de Zarate, J. D. (2016). La formulación magistral del siglo XXI. Panorama actual del medicamento, 40(392), 106-111.

PwC, P. C. (2011). Pharma 2020: Supplying the future Which path will you take? Londres: Pharmaceuticals and Life Sciences. Obtenido de www.pwc.com/pharma2020

Rankings, Q. W. (2020). Pharmacy and Pharmacology, h. Recuperado el 07 de 04 de 2021, de https://www.topuniversities.com/university-rankings/universitysubject-rankings/2018/pharmacy-pharmacology

Rodríguez, A. (2012). Perspectivas y retos de la Formulación magistral. RevistaAcofar, 1(14), 1-12.

Rodríguez-Rodríguez, J., \& Reguant-Álvarez, M. (2020). Calcular la fiabilidad de un cuestionario o escala mediante el SPSS: el coeficiente alfa Cronbach. REIRE. Revista d'Innovació $i$ Recerca en Educació, 13(2), 1-13. doi:https://doi.org/10.1344/reire2020.13.230048

Ruiz, M. (17 de 07 de 2015). La prescripcion en formulacion Magistral Dermatologica. Propuesta de receta medica. Revisata ACOFAR. Recuperado el 23 de 03 de 2021, de https://revistaacofar.com/mi-farmacia/formulacion-magistral/la-prescripcionen-formulacion-magistral-dermatologica-propuesta-de-receta-medica/

Sacoto, A. K. (2 de 12 de 2016). Relación entre la dispensación del servicio de farmacia aprovisionada con medicamentos de la 9na. revisión del cuadro nacional de medicamentos básicos, y la prescripción de los especialistas del Hospital Dermatológico Gonzalo González. Quito: Colegio de Posgrados Universidad San Francisco de Quito.

Vite-Vera, F., \& Párraga-Fernández, J. (2019). Análisis de la concentración de mercado del sector de elaboración de sustancias farmacéuticas en Ecuador. Período 20102017. (E. Ortiz-Prado, Ed.) X-Pedientes Económicos, 3(5), 6-16. 
Zebroski, R. (2016). A Brief History of Pharmacy (en linea). New York, USA: Routledge. Recuperado el 23 de 06 de 2018, de https://sci-hub.tw/10.4324/9781315685830

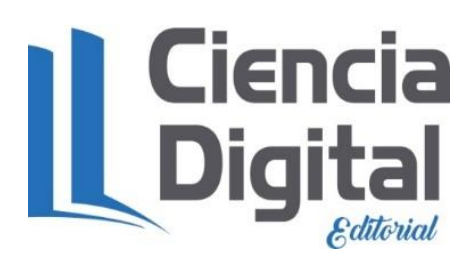




\section{PARA CITAR EL ARTÍCULO INDEXADO.}

Guananga Diaz, N. I., Rodríguez Vinueza, V. I., Cando Brito, V. M., \& Escobar Torres, J. A. (2021). Médicos y bioquímicos farmacéuticos en el cuidado personalizado de la salud con la formulación magistral. Anatomía Digital, 4(2), 99-121. https://doi.org/10.33262/anatomiadigital.v4i2.1668

\section{Ciencia \\ LDigital}

El artículo que se publica es de exclusiva responsabilidad de los autores y no necesariamente reflejan el pensamiento de la Revista Anatomía Digital.

El artículo queda en propiedad de la revista y, por tanto, su publicación parcial y/o total en otro medio tiene que ser autorizado por el director de la Revista Anatomía Digital.
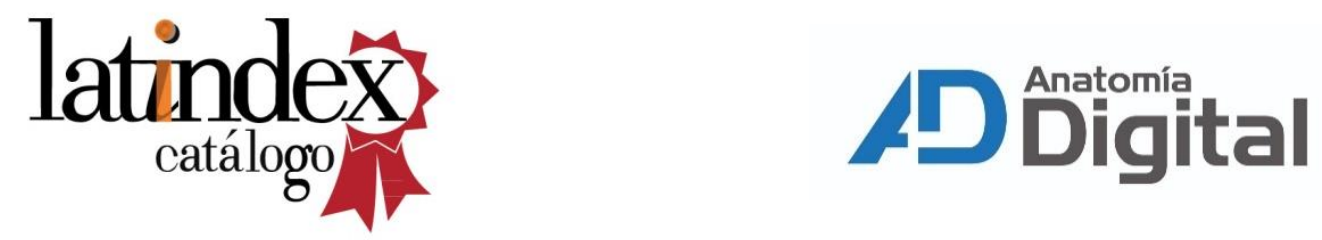